\title{
RELATO DE CASO: DESCRIÇÃO DA EVOLUÇÃO DA COMUNICAÇÃO ALTERNATIVA NA PRAGMÁTICA DO ADULTO PORTADOR DE AUTISMO
}

\section{Case report: describing the development of alternative communication on the adult autism bearer pragmatic}

\author{
Patrícia Reis Ferreira ${ }^{(1)}$, Eny Viviane da Silva Teixeira ${ }^{(2)}$, \\ Denise Brandão de Oliveira e Britto ${ }^{(3)}$
}

\begin{abstract}
RESUMO
Tema: alguns pacientes portadores de autismo necessitam do apoio de métodos alternativos de comunicação para se interagir e comunicar de forma eficaz. A comunicação alternativa e/ou suplementar (CAS) promove possibilidades comunicativas através da utilização integrada de símbolos, recursos, estratégias e técnicas. Procedimentos: os objetivos deste estudo foram descrever os efeitos da utilização simultânea de dois métodos alternativos de comunicação, para a ampliação das habilidades pragmáticas de um adulto portador de autismo. A pesquisa é um estudo de caso do tipo longitudinal, de um indivíduo de 20 anos, diagnósticado com autismo. Os recursos comunicativos utilizados pelo sujeito foram avaliados, por meio da prova de pragmática do teste de linguagem infantil ABFW, antes e depois da utilização, ao longo de nove meses, dos métodos de comunicação alternativa: PECS Adaptado e Fala Sinalizada. Os dados coletados antes e após o uso dos recursos da comunicação alternativa foram comparados quali-quantitativamente. Resultados: os dados coletados mostraram que houve aumento do número de atos comunicativos e funções comunicativas, e que o percentual do espaço comunicativo ocupado pelo sujeito aumentou após os procedimentos realizados com o uso dos programas de comunicação alternativa. Conclusão: concluiu-se que houve progresso no perfil pragmático da comunicação com o uso concomitante dos dois métodos de comunicação alternativa, uma vez que as interações sociais do sujeito aumentaram.
\end{abstract}

DESCRITORES: Linguagem; Estudos de Linguagem; Transtornos da Linguagem; Auxiliares de Comunicação para Deficientes

(1) Fonoaudióloga do Consultório de Fonoaudiologia e Psicologia, Belo Horizonte, MG; Especialista em Linguagem pelo CEFAC - Pós-Graduação em Saúde e Educação, Belo Horizonte, MG.

(2) Fonoaudióloga da Companhia Siderúrgica Nacional; Especializanda em Linguagem pelo CEFAC - Pós-Graduação em Saúde e Educação, Belo Horizonte, MG.

(3) Fonoaudióloga; Docente na área de Linguagem no curso de Fonoaudiologia da Pontifícia Universidade Católica, PUC, Belo Horizonte, MG; e de Metodologia Científica no CEFAC - Pós-Graduação em Saúde e Educação, Belo Horizonte, MG; Mestre em Engenharia de Produção pela Universidade Federal de Santa Catarina; Doutoranda em Língua Portuguesa e Linguística pela Pontifícia Universidade Católica de Minas Gerais.

Conflito de interesses: inexistente

\section{INTRODUÇÃO}

O diagnóstico de autismo conta hoje, com vários instrumentos, os quais a maior parte deles avalia a interação social, a comunicação e os padrões restritos e repetitivos de comportamento, interesses e atividades ${ }^{1}$. No entanto, existe ainda, a necessidade de critérios diagnósticos mais precisos e apropriados $^{2,3}$, para que seja possível uma intervenção precoce e individualizada ${ }^{4}$. Alguns estudos estão sendo realizados neste sentido e observa-se que as dificuldades de comunicação podem ser elementos importantes para o diagnóstico precoce ${ }^{5}$. 
Um estudo aponta que os aspectos funcionais da comunicação devem ser avaliados, pois os mesmos apresentam-se prejudicados no autismo ${ }^{6}$. Outro, mostra o perfil da relação entre função e aquisição de linguagem, a partir de relatos familiares ${ }^{7}$, os quais apresentam um grande valor, pois contribuem como material para análise da comunicação funcional, logo facilitam o diagnóstico.

Para quantificar tais aspectos funcionais da comunicação, utiliza-se como unidade mínima de análise, os atos comunicativos. Estes podem ser expressos pelos meios verbal e não verbal. O primeiro ocorre quando pelo menos $75 \%$ dos fonemas da língua são falados, e o último é dividido em vocal, que abrange todas as outras emissões, e gestual, que envolve movimentos do corpo e do rosto. Os atos comunicativos iniciam-se quando ocorre a interação entre o adulto e uma criança ou entre uma criança e um objeto, e terminam quando o foco de atenção da criança muda ou há troca de turno ${ }^{6}$.

Além disso, os atos são analisados através de 20 categorias funcionais, sendo elas: pedido de objeto, pedido de ação, pedido de rotina social, pedido de consentimento, pedido de informação, protesto, reconhecimento do outro, exibição, comentário, auto-regulatório, nomeação, performativo, exclamativo, reativo, não focalizada, jogo, exploratória, narrativa, expressão de protesto e jogo compartilhado ${ }^{6}$. Pretende-se através destes aspectos, compreender o desenvolvimento de linguagem relacionando as características comunicativas ao quadro clínico ${ }^{9}$.

Uma pesquisa observou que há um equilíbrio na quantidade de atos comunicativos expressos por minuto, na interação entre adulto e crianças de quatro e cinco anos de idade, com o desenvolvimento normal, ainda que tenha sido encontrada diferença estatisticamente significativa com relação a essa medida ${ }^{8}$.

Devido a um déficit na comunicação, algumas pessoas com autismo necessitam da utilização de Comunicação Alternativa e/ou Suplementar (CAS), pois quando apresentam a fala, geralmente esta não é funcional, às vezes muito ecolálica, ou com emissões vocálicas desprovidas de sentido, entre outros. A CAS promove possibilidades comunicativas para essas pessoas, através da utilização integrada de símbolos, recursos, estratégias e técnicas ${ }^{10}$. Diz-se que a comunicação é suplementar quando o usuário a utiliza como apoio, apenas para complementar a comunicação oral, sem substituí-la. E ela é alternativa, quando por alguma impossibilidade articulatória ou de produção de sons, a pessoa utiliza somente outro meio de comunicação, ao invés da fala ${ }^{12}$, sendo que qualquer método de comunicação pode ser utilizado como alternativo ou suplementar.

Várias são as maneiras de se utilizar uma Comunicação Alternativa e/ou Suplementar, sendo eleita através da adequação para cada sujeito, como por exemplo, o nível de cognição, a atenção visual, a intenção comunicativa, destreza manual, entre outros. Aqui, serão descritos apenas os métodos Fala Sinalizada, e The Picture Exchange Communication Symbols (PECS).

A Fala Sinalizada é um programa que foi desenvolvido para pessoas com necessidades especiais que apresentam graves alterações de linguagem e comunicação. Ele consiste no uso simultâneo da fala e de um gesto correspondente, que pode ser uma adaptação do sinal utilizado na Língua de Sinais. Vale ressaltar, que a Língua de Sinais propriamente dita, segue uma estrutura própria e não necessariamente vem acompanhada pela fala. Já o sistema de Fala Sinalizada, segue a estrutura da linguagem oral não sendo, pois, considerado como Língua de Sinais. O objetivo deste programa, é que inicialmente, a pessoa se comunique espontaneamente através dos sinais manuais, depois utilize os sinais concomitantemente à fala, e posteriormente evolua para a linguagem verbal espontânea e generalizada para todos os ambientes ${ }^{11}$.

O PECS é um método, desenvolvido para pessoas com autismo e outros transtornos da comunicação, que utiliza objetos concretos, miniaturas, fotos, e/ou pictogramas, para estabelecer a comunicação através da troca, ou seja, a pessoa entrega um cartão simbolizando o que deseja, e recebe aquilo que solicitou. Este método visa auxiliar pessoas que não têm intenção comunicativa, como por exemplo, os autistas, pois trabalha a intenção exigindo a aproximação do outro, seguida de uma solicitação, estabelecendo assim a comunicação ${ }^{12}$.

Em uma dissertação de pós-graduação em educação especial, descreveu-se sobre os efeitos do PECS adaptado ao Currículo Funcional Natural, em pessoas com autismo infantil ${ }^{12}$. O Currículo Funcional Natural é uma metodologia educacional que prioriza a independência máxima do aluno nas atividades de vida diária, através de um currículo desenvolvido no ambiente natural e de forma funcional. Tem por objetivo "ensinar conhecimentos e habilidades que possam ser usadas pelo aluno, sendo úteis em diferentes ambientes e que possam continuar sendo úteis através do tempo" ${ }^{13}$. A autora da dissertação concluiu que o PECS-adaptado por ser de fácil compreensão, clareza e utilidade na obtenção do item desejado, pode ser utilizado no ambiente natural de cada pessoa, e contribui tanto 
para o processo de comunicação, como também na qualidade de vida das pessoas com autismo ${ }^{12}$.

Tendo como base os métodos de CAS, pensouse na fusão de dois deles, com o intuito de desenvolver a pragmática de uma pessoa portadora de autismo, pois, se um método não tem correspondido às expectativas, segundo seu avanço em relação a novas aquisições e a funcionalidade do uso, porque não utilizar um segundo método sem deixar de estimular o que foi iniciado primeiro? Isso seria fator de confusão para a aprendizagem dos métodos, ou mais uma opção de comunicação, na qual o sujeito poderia lançar mão do método mais prático e mais eficaz naquele determinado momento, e com aquele determinado interlocutor?

A partir dos argumentos expostos, este trabalho tem como objetivo principal descrever o efeito da utilização concomitante de dois métodos de comunicação alternativa, para a ampliação das habilidades pragmáticas de um adulto portador de autismo

\section{APRESENTAÇÃO DO CASO}

O presente estudo de caso é do tipo longitudinal, e descreve sobre um sujeito nomeado B., sexo masculino, 20 anos, e com o diagnóstico de autismo. Ele não possui comunicação verbal estabelecida.

De acordo com os dados do prontuário da escola que B estuda, a gestação foi planejada e desejada, no entanto, durante a gravidez, houve entre os pais, um período de muita briga e ansiedade.

Segundo a mãe, aos oito meses o olhar de B. era diferente, o bebê não interagia, e depois que começou a andar, a linguagem regrediu. Aos dois anos, chorava muito e girava objetos. Em torno dos 3 anos de idade, a mãe o levou ao fonoaudiólogo, pois suspeitava de surdez, já que a criança não falava. Este o encaminhou ao otorrino para descartar tal hipótese. Ao retornar ao consultório, o fonoaudiólogo com a confirmação de que B. não era surdo, e suspeitando de autismo, o encaminhou a uma psicóloga que concluiu o diagnóstico.

Com o passar dos anos, seu desenvolvimento foi acompanhado por diversos profissionais como psicólogos, psiquiatras e neurologistas.

$\mathrm{Na}$ época em que foi realizada a pesquisa, B. fazia uso dos medicamentos, Trileptal e Depakene que são anticonvulsivantes, e Rivotril que é um tranquilizante.

Sua linguagem referia-se apenas à situação imediata e concreta, e não respondia sistematicamente a uma solicitação. Apresentava dificuldade de compreensão de ordens, de concentração e atenção visual. Demonstrava pouca intenção comunicativa, utilizando-se de meios vocais e gestuais para estabecer interação. Possuía um déficit significativo da linguagem expressiva quanto aos aspectos de semântica, sintaxe, prosódia e fonologia caracterizando-se como ausência de fala funcional.

A pesquisa foi realizada no $\mathrm{CEFAC}-\mathrm{BH}$, sendo que tanto os procedimentos quanto os dados coletados ocorreram na instituição "Cooperativa de Educação Especial Dia Dia", que se localiza na Rua Halley, 408 - Santa Lúcia, CEP 30360-330, Belo Horizonte - MG. Foram priorizados dois métodos de comunicação alternativa: The Picture Exchange Communication System adaptado ao Currículo Funcional Natural (PECS - Adaptado) ${ }^{12}$, e a Fala Sinalizada ${ }^{11}$.

Tais métodos foram eleitos, pois $\mathrm{B}$. já utilizava a fala sinalizada em situação de treino, desde 1999 e vinha, demonstrando pequenos avanços neste método, o que justificava a não retirada deste para introdução de novos métodos. O PECS foi selecionado por ser um sistema de comunicação que exige a troca do cartão, logo, estimula a intenção em se comunicar, que era uma habilidade muito pouco desenvolvida, além de exigir uma grande atenção visual, o que normalmente não era exigido do aluno, e, portanto, apresentava dificuldade. Como a instituição onde estuda adota o Currículo Funcional Natural como metodologia educacional, optou-se por utilizar o PECS - Adaptado.

Quando se iniciou esta pesquisa, B. solicitava três itens de seu interesse, apenas em situação de treino, através da fala sinalizada. Segundo relatos da instituição, B. permaneceu por muito tempo apenas com o primeiro sinal, até que compreendesse a proposta do método. A partir do início deste estudo, ou seja, da primeira avaliação pragmática, foram utilizados os dois métodos concomitantes, partindo do princípio que o PECS - Adaptado deveria ser priorizado para aqueles itens em que o sinal utilizado na LIBRAS fosse de difícil adaptação ao método, ou para aqueles sinais com os quais o sujeito da pesquisa não obtinha sucesso com o método da Fala Sinalizada. A seleção dos símbolos foi feita a partir dos interesses e necessidades do aluno, baseado nos relatos da mãe e na observação dos profissionais da instituição.

$\mathrm{Na}$ sala em que passava a maior parte do período em que se encontrava na escola, havia um painel de comunicação com diversos símbolos, representados através de pictograma, objeto real e foto, uma vez que os alunos da sala se encontravam em níveis de compreensão simbólica diversificado.

A estimulação de ambos os métodos, ou seja, o treino formal, ocorreu de segunda a sexta-feira, com duração de 20 minutos para cada sistema de comunicação, salvo quando B. demonstrava cansaço ou 
por algum motivo se recusava a dar continuidade ao treinamento. Nesta estimulação estavam presentes outros alunos da escola, realizando a mesma atividade, exceto quando o método estava em fases nas quais era exigido um ambiente controlado. Além desses 40 minutos de treinamento, o sujeito era estimulado a utilizar os métodos informalmente, ao longo das 4 horas diárias que se encontrava na instituição, auxiliando na generalização da comunicação. Neste período também eram desenvolvidas, por uma das pesquisadoras, atividades que exigiam atenção visual, atenção auditiva, reconhecimento de pictogramas, concentração, interação social, contato ocular com profissionais e colegas, localização espacial, e atividade de vida prática e diária associada ao uso de pictogramas.

O protocolo de avaliação utilizado foi a prova de pragmática do ABFW - Teste de Linguagem Infantil nas Áreas de Fonologia, Vocabulário, Fluência e Pragmática ${ }^{6}$.

Os dados foram transcritos para a ficha de análise dos aspectos funcionais da comunicação (Figura 1), retirada do teste ${ }^{6}$, para serem analisados posteriormente.

A coleta de dados sugerida pelo teste é uma filmagem de 30 minutos consecutivos, de interação em forma de brincadeira com um adulto familiar. No entanto, esta foi realizada em situação de interação social, durante 23 minutos não consecutivos, pois, levando-se em conta que o indivíduo da pesquisa é um adulto, pôde-se aproveitar variados contextos para tal avaliação, e não apenas a interação em forma de brincadeira. O motivo de ter sido filmado por um período menor que o sugerido no teste, foi devido à preocupação constante de retirar $\mathrm{B}$. o mínimo possível de sua rotina diária, uma vez que se sabe que esta é muito importante para a sua organização. Ambos os registros foram realizados em momentos como o lanche, o recreio, escovação de dentes e interação livre entre os colegas da sala e a pesquisadora, sendo, portanto, as duas filmagens, passíveis de comparação.

A avaliação foi realizada em dois momentos. A primeira análise a partir de uma filmagem em março de 2006, e a última, no final do ano, após 9 meses de treinamento com os dois métodos de comunicação.

A participação de B. na pesquisa ocorreu por meio da autorização do responsável que assinou o termo de consentimento livre e esclarecido e autorização de fotos e filmagens.

Este estudo foi avaliado pelo Comitê de Ética em Pesquisa (CEP) do CEFAC - Pós-Graduação em Saúde e Educação, e aprovado pelo protocolo 123/06.
Os dados coletados são apresentados de forma descritiva com freqüência absoluta (números absolutos) e relativa (percentuais).

\section{RESULTADOS}

O processo inicial da introdução dos dois sistemas de Comunicação Alternativa concomitantemente foi composto pelas seguintes etapas: apresentação do objeto, correlação do objeto com o símbolo e introdução do aluno ao uso do sistema.

B. demonstrou confusão em alguns momentos da estimulação, em relação a qual sinal ou cartão deveria ser utilizado, principalmente quando era introduzido um novo objeto de desejo. Em resposta a esse comportamento, o cartão ou sinal era retirado temporariamente, com o intuito de trabalhar melhor os anteriores, e a correlação do objeto com o símbolo novo.

Algumas das características do aluno que dificultaram a introdução dos sistemas de comunicação alternativa foram a dificuldade de concentração, a agitação, e a dificuldade de atenção visual, que diminuíram no decorrer das estimulações propostas pela pesquisa, uma vez que essas habilidades vinham sendo trabalhadas diariamente.

Analisando os dados ilustrados na Figura 2, constatou-se que na primeira amostra, o número total de atos comunicativos expressos pelo sujeito da pesquisa foi de apenas 2 (dois), ao longo de toda a filmagem, sendo o número de atos comunicativos expressos por minuto igual a 0,086. Este número é calculado através da divisão do número de atos comunicativos pelo tempo de amostra. Dentre os atos foi utilizada somente a função de protesto, sendo as duas vezes por meio gestual. Quanto ao espaço comunicativo, que mostra a participação de um indivíduo na interação, observou-se que o percentual ocupado por B. foi de 18,18\%.

$\mathrm{Na}$ segunda amostra, Figura 3 , o número total de atos comunicativos expressos pelo sujeito da pesquisa foi de 12 (doze) atos, sendo sete vezes através da função de pedido de objeto, pelo meio gestual e vocal concomitantemente; três vezes por meio de pedido de ação através do meio gestual; e uma vez com a função de reconhecimento do outro, utilizando também o meio gestual. O número de atos comunicativos expressos por minuto foi igual a 1,91 e o percentual do espaço comunicativo ocupado por ele foi de $52,17 \%$.

Não foi possível quantificar o número de funções não focalizadas realizadas pelo indivíduo, uma vez que ele executa balanceios, vocalizações e gestos estereotipados constantemente. No entanto percebe-se nitidamente a diminuição destes 


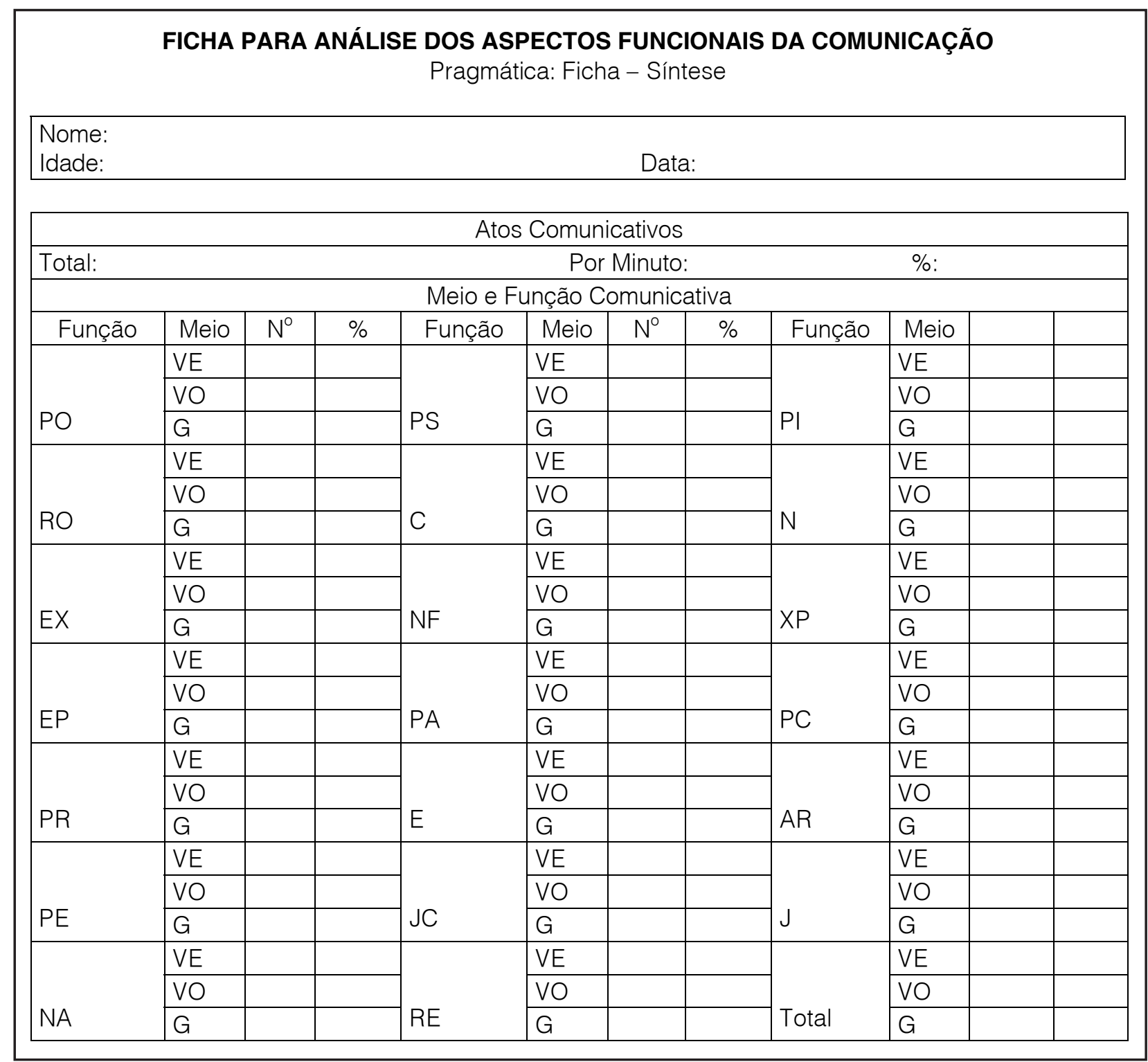

Função: função comunicativa

Meio: meio comunicativo

No: número de atos comunicativos

$\%$ : porcentagem de meios comunicativos

VE: meio comunicativo verbal

VO: meio comunicativo vocal

G: meio comunicativo gestual

PO: pedido de objeto

RO: reconhecimento do outro

EX: exclamativo

EP: expressão de protesto

PR: protesto

PE: performativo

NA: narrativa
PS: pedido de rotina social

C: comentário

NF: não-focalizada

PA: pedido de ação

E: exibição

JC: jogo compartilhado

RE: reativos

PI: pedido de informação

$\mathrm{N}$ : nomeação

XP: exploratório

PC: pedido de consentimento

AR: auto-regulatório

$\mathrm{J}$ : jogo

Figura 1 - Ficha para análise dos aspectos funcionais da comunicação - amostra realizada com o sujeito da pesquisa antes da estimulação 
Idade: 20 ANOS

Data: 24/03/2006

\begin{tabular}{|c|c|c|}
\hline Atos Comunicativos & & \\
\hline Total: 2 & Por Minuto:0,086 & $\%: 18,18$ \\
\hline
\end{tabular}

\begin{tabular}{|c|c|c|c|c|c|c|c|}
\hline Função & Meio & $\mathrm{N}^{\circ}$ & $\%$ & Função & Meio & $\mathrm{N}^{\circ}$ & $\%$ \\
\hline \multirow{3}{*}{ PR } & $\mathrm{VE}$ & 0 & 0 & \multirow{3}{*}{ Total } & VE & 0 & 0 \\
\hline & VO & 0 & 0 & & VO & 0 & 0 \\
\hline & $G$ & 2 & 100 & & $G$ & 2 & 100 \\
\hline
\end{tabular}

Função: função comunicativa

Meio: meio comunicativo

No: número de atos comunicativos

$\%$ : porcentagem de meios comunicativos

PR: protesto

VE: meio comunicativo verbal

VO: meio comunicativo vocal

G: meio comunicativo gestual

Fonte: Fernandes F D M. Pragmática. In: Andrade C R F, Befi-Lopes D M, Fernandes F D M, Wertzner H F. ABFW - Teste de Linguagem Infantil nas áreas de fonologia, vocabulário, fluência e pragmática. Pró-fono departamento editorial. Carapicuíba SP 2000. pág 88.

Figura 2 - Ficha para análise dos aspectos funcionais da comunicação - amostra realizada com o sujeito da pesquisa antes da estimulação

\begin{tabular}{|c|c|c|c|c|c|c|c|c|c|c|c|}
\hline \multicolumn{5}{|c|}{ Idade: 20 ANOS } & \multicolumn{7}{|c|}{ Data:22/12/2006 } \\
\hline \multicolumn{12}{|c|}{ Atos Comunicativos } \\
\hline \multicolumn{4}{|c|}{ Total: 12} & \multicolumn{4}{|c|}{ Por Minuto:1,91 } & \multicolumn{4}{|c|}{$\%: 52,17$} \\
\hline \multicolumn{12}{|c|}{ Meio e Função Comunicativa } \\
\hline Função & Meio & $\mathrm{N}^{0}$ & $\%$ & Função & Meio & $\mathrm{N}^{0}$ & $\%$ & Função & Meio & $\mathrm{N}^{0}$ & $\%$ \\
\hline \multirow{3}{*}{$\mathrm{PO}$} & VE & 0 & 0 & \multirow{3}{*}{ PA } & VE & 0 & 0 & \multirow{3}{*}{$\mathrm{RO}$} & VE & 0 & 0 \\
\hline & $\mathrm{VO}$ & 1 & 12,5 & & $\mathrm{VO}$ & 0 & 0 & & $\mathrm{VO}$ & 0 & 0 \\
\hline & $G$ & 7 & 87,5 & & $G$ & 3 & 100 & & $G$ & 1 & 100 \\
\hline \multirow{3}{*}{ Total } & VE & 0 & 0 & & & & & & & & \\
\hline & VO & 1 & 0,91 & & & & & & & & \\
\hline & $G$ & 11 & 91,66 & & & & & & & & \\
\hline
\end{tabular}

Função: função comunicativa

Meio: meio comunicativo

No: número de atos comunicativos

$\%$ : porcentagem de meios comunicativos

PR: protesto

PO: pedido de objeto

PA: pedido de ação

RO: reconhecimento do outro

VE: meio comunicativo verbal

VO: meio comunicativo vocal

G: meio comunicativo gestual

Fonte: Fernandes F D M. Pragmática. In: Andrade C R F, Befi-Lopes D M, Fernandes F D M, Wertzner H F. ABFW - Teste de Linguagem Infantil nas áreas de fonologia, vocabulário, fluência e pragmática. Pró-fono departamento editorial. Carapicuíba SP 2000. pág 88.

Figura 3 - Ficha para análise dos aspectos funcionais da comunicação - amostra realizada com o sujeito da pesquisa após a estimulação

Rev. CEFAC. 2011 Mai-Jun; 13(3):559-567 
comportamentos no decorrer da pesquisa e ao comparar os dois registros.

Ao longo da pesquisa, foram acrescentados gradativamente os sinais e os pictogramas. No total, foram ensinados três sinais, além dos que ele já conhecia, e três pictogramas. Um dos sinais, ele não conseguiu reproduzir, sendo então trabalhado na metodologia do PECS.

Em um momento de necessidade, o aluno utilizou um pictograma do quadro de comunicação da sala, para solicitar o que desejava, ao invés de usar sinal que já havia aprendido para aquele signo. Este pictograma foi trabalhado anteriormente apenas no emparelhamento de símbolos iguais, sem ser correlacionado ao objeto real, como é o processo usual de introdução de símbolos. Então após este episódio, o pictograma foi incluído em seu álbum de comunicação, para que B. pudesse utilizar o método que mais se adequasse ao momento.

$O$ aluno demonstrou ter iniciado o processo de generalização da comunicação uma vez que em alguns momentos, solicitou, em ambientes variados e de forma espontânea através da comunicação alternativa, objetos de seu interesse.

\section{DISCUSSÃO}

O número de atos comunicativos expressos por minuto varia de indivíduo para individuo, levandose em consideração os interlocutores e situações apresentadas ${ }^{6}$. No entanto, pesquisas desenvolvidas até 0 atual momento ${ }^{6}$ revelam algum parâmetro desses atos por minuto, segundo a tabela abaixo:

\section{TABELA 1 - Relação aproximada de atos comunicativos em pessoas normais}

\begin{tabular}{llllllll}
\hline $\begin{array}{l}\text { Idade aproximada } \\
\text { (meses) }\end{array}$ & 1 & 3 & 12 & 30 & 60 & 90 & adultos \\
\hline $\begin{array}{l}\text { Atos comunicativos } \\
\text { (por minutos) }\end{array}$ & 1 & 2 & 3 & 6 & 8 & 9 & 10 \\
\hline
\end{tabular}

Fonte: Fernandes F D M. Pragmática. In: Andrade C R F, Befi-Lopes D M, Fernandes F D M, Wertzner H F. ABFW - Teste de Linguagem Infantil nas áreas de fonologia, vocabulário, fluência e pragmática. Pró-fono departamento editorial. Carapicuíba SP 2000. pág 84 .

Observa-se que a discrepância existente entre o indivíduo da pesquisa e os dados desta tabela se atribui ao fato do mesmo apresentar transtorno global do desenvolvimento (autismo), uma vez que este é caracterizado por ausência de interações comunicativas e sociais; comportamento focalizado e/ou repetitivo; fobias; perturbações do sono e ou alimentação; crises; birras; e, agressividades ${ }^{1}$.

Este menor número de atos comunicativos também foi encontrado em estudos comparando crianças normais e crianças com alterações no desenvolvimento de linguagem ${ }^{14}$.

Um estudo realizado com indivíduos de 3 a 14 anos, utilizando 54 sujeitos portadores de autismo, encontrou uma média de 2,66 atos por minuto, o que não se mostra discrepante de nossa última amostra, que observou 1,91 atos comunicativos por minuto, levando-se em conta que aquele resultado é uma média ${ }^{15}$.

Sabe-se que em crianças com autismo o maior número de atos comunicativos por minuto está relacionado ao aumento no uso de comunicação oral (verbalizações e vocalizações), contudo a maior parte dos atos comunicativos expressos por um autista é pelo meio gestual ${ }^{15}$, o que está em concordância com esta pesquisa, uma vez que o indivíduo em estudo realizou todos os atos comunicativos através de gestos, e em apenas um, utilizou vocalização concomitante ao gesto.

Neste estudo, observa-se que as funções de maior ocorrência foram de protesto e de pedidos de objeto e de ação, o que concorda com uma pesquisa, que relata a presença de comunicação espontânea em crianças autistas, afirmando que as primeiras funções a emergir são estas, pois são usadas para regular o comportamento de outra pessoa para obter algo desejado ${ }^{16}$.

A diferença relacionada à porcentagem dos atos comunicativos das duas amostras, retrata um aumento representativo das interações comunicativas, considerando que a relação mais simétrica entre os interlocutores auxilia no desenvolvimento das habilidades pragmáticas ${ }^{17}$, e que a unidade mínima de análise deixa de ser o fonema, o vocábulo ou frase e passa a ser o ato comunicativo, que será utilizado para a análise funcional da comunicação ${ }^{6,15}$.

Levando em consideração as duas amostras, percebe-se a diminuição da agitação do sujeito da pesquisa, que pode estar relacionada ao aumento da habilidade comunicativa. No entanto essa diminuição deste comportamento também pode 
ser resultado de outros fatores, como emocional, mudança ou não de rotina, medicação adequada ou não, etc., não sendo possível então, atribuir a essa menor agitação, apenas o fator comunicativo.

Diante de observações do comportamento do sujeito, realizadas ao longo desta pesquisa, como por exemplo, a maior interação espontânea com os colegas e profissionais da instituição que frequenta, e dos dados coletados, é possível notar que neste estudo, houve melhora na pragmática com a utilização dos dois métodos de comunicação alternativa (PECS-adaptado e Fala Sinalizada), até o presente momento. No entanto, percebe-se que o período de 9 meses, utilizado para realizar esta pesquisa, foi muito curto, uma vez que se trata de um público no qual as melhoras conquistadas são observadas após um período maior de trabalho.

Poderia ser questionado se o sucesso da pesquisa foi devido ao fato do método utilizado anteriormente (Fala Sinalizada) não estar adequado às necessidades do aluno, no entanto, durante todo esse período, o indivíduo demonstrou maior interesse pela Fala Sinalizada, o que mostra uma identificação positiva com este sistema, sugerindo sua adequação. Além disso, o sujeito da pesquisa demonstra facilidade em executar sinais, e pouca atenção visual, mesmo tendo evoluído nesta habilidade, o que dificulta a utilização do PECS-adaptado e facilita a da fala sinalizada, tornando-a mais apropriada.

Outro fato a ser observado, é que a estimulação da atenção visual, da atenção ao outro e da iniciativa de comunicação exigidas pelo PECS-adaptado, desenvolveu no sujeito da pesquisa habilidades não priorizadas anteriormente, o que pode ter auxiliado em aspectos da pragmática, como nas funções comunicativas de pedido de objeto e ação, reconhecimento do outro e na interação com o outro. Portanto, aliar estes dois métodos, pôde complementar as necessidades do sujeito em questão.

Sugere-se que o estudo seja feito por um período maior, com o intuito de obter mais dados quantitativos e qualitativos da utilização de métodos concomitante com o público autista.

\section{CONCLUSÃO}

A identificação de variações sutis permite concluir, apesar do curto período de tempo, que houve progresso no perfil pragmático da comunicação, com o uso dos dois métodos de comunicação alternativa, uma vez que as interações interpessoais e sociais aumentaram, e o espaço comunicativo se equilibrou.

Um fator importante a ser observado é que o uso concomitante de dois métodos de Comunicação Alternativa, não deve ser aplicado a qualquer portador de autismo, uma vez que é necessário valorizar as habilidades de cada indivíduo, minimizando suas dificuldades.

Os dados que irão nortear sobre qual intervenção, ou sistema de comunicação será mais adequado, dizem respeito à capacidade cognitiva, à destreza manual, à intenção comunicativa, à capacidade de simbolização e à acuidade visual, associados às relações interpessoais e sociais, e aos demais aspectos do desenvolvimento.

\begin{abstract}
Background: some autism patients need the support of alternative communication methods in order to interact and communicate in an efficient manner. The alternative and/or supplementary communication promotes communicative means through the integrated use of symbols, resources, strategies and techniques. Procedures: the purpose of this study is to describe the effects of the simultaneous application of two alternative communication methods on autistic adults. This research is a longitudinal type case study of a 20 year old individual diagnosed with autism. The communicative resources used by the individual were evaluated by applying the child language pragmatic ABFW test, during nine months, by using the following alternative communication methods: PECS - Adapted and Signalized Speech. Data collected before and after applying the alternative communication resources methods were compared under qualitative and quantitative approaches. Results: the collected data have shown an increase in the number of communicative acts and functions, as well as an increase in the communicative space occupied by the individual, after the procedures that were applied by using the alternative communication programs. Conclusion: as a conclusion we perceived the achievement of progress in the communication pragmatic profile by concomitantly using both alternative communication methods, once that the individual's social interactions increased.
\end{abstract}

KEYWORDS: Language; Language Arts; Language Disorders; Communications Aids for Disabled 


\section{REFERÊNCIAS}

1. Lampreia C. Avaliações quantitativa e qualitativa de um menino autista: uma análise crítica. Psicol. estud., Maringá, [online]. v.8, n. 1, June 2003. [acesso em 22 Set. 2009]. Disponível em: <http:// www.scielo.br/scielo.php?script=sci_arttext\&pid= S1413-73722003000100008\&lng=en\&nrm=iso $>$.

2. Lampreia C. Perspectivas da pesquisa prospectiva com bebês irmãos de autistas. Psicol. cienc. aprof., mar. 2009; 29 (1):160-71.

3. Orsmond GI, Krauss MW, Setzer MM. Peer relationships and social and recreational activities among adolescents and adults with autism. J Autism Dev Disord. 2004; 34(3):245-56.

4. Lampreia C. A perspectiva desenvolvimentista para a intervenção precoce no autismo. Estudos de Psicologia. Jan.-mar. 2007; 24(1):105-14.

5. Granz JB, Simpson RL. Effects on communicative requesting and speech development of the Picture Exchange Communication System in children with characteristics of autism. J Autism Dev Disord. 2004; 34(4):395-409.

6. Andrade CRF, Befi-Lopes DM, Fernandes FDM, Wertzner HF. ABFW - Teste de Linguagem Infantil nas áreas de fonologia, vocabulário, fluência e pragmática. Carapicuíba: Pró-Fono; 2000.

7. Charman T, Howlin P, Berry B, Prince E. Measuring developmental progress of children with autism spectrum disorder on school entry using parent report. Autism. 2004; 8(1):89-100.

8. CERVONE, L. M.; FERNANDES, F. D. M. Análise do perfil comunicativo de crianças de 4 e 5 anos na interação com o adulto. R. Soc. Bras. Fonoaudiol., São Paulo, 2005;10(2):97-105.

9. De Paula KMP; Enumo SRF. Avaliação assistida e comunicação alternativa: procedimentos para a educação inclusiva. Rev. bras. educ. espec., Marília. [online]. 2007; 13(1). [acesso em: 22 set. 2009]. Disponível em: <http://www. scielo.br/scielo.php?script=sci_arttext\&pid= S1413-65382007000100002\&lng=en\&nrm=iso > .

10. Chun RY. O desenvolvimento da comunicação não-verbal através dos símbolos Bliss em indivíduo não falante portador de paralisia cerebral. Dist Comun. Out 1991; 4:121-36.

11. 17.Schaeffer B, Musil A, Kollinzas G. Total communication: asigned speech program for non-verbal children. Campaing: Illionois:Resarch Press; 1980.

12. Walter CCF. Os efeitos da adaptação do PECS associada ao Curriculum funcional em pessoas com autismo infantil [dissertação]. São Carlos (SP): Universidade Federal de São Carlos; 2000.

13. LeBlanc JM, Mayo L. Centro Ann Sullivan del Peru para la integracion a la vida de las personas con discapacidade severas. Boletin del Real Patronato. 1999; 44:24-39.

14. Rodrigues A, Befi-lopes DM. Comparação entre as habilidades pragmáticas de crianças normais e crianças com alteração de desenvolvimento de linguagem. Rev. Soc. Bras. Fonoaudiol. 2004; 9(2):81-7.

15. Fernandes FDM. Perfil da pragmática de sujeitos com quadro psiquiátricos na primeira avaliação fonoaudiológica. Rev Soc Bras Fonoaudiol. 2002; 7(1):38-43.

16. Stone WL, Caro-Martinez LM. Naturalistic observations of spontaneous communication in autistic children. J Autism Dev Disord. 1990; 20(4):437-57.

17. Fernandes FDM. Sugestões de procedimentos terapêuticos de linguagem em distúrbios de espectro autístico. In: Limongi SCO, organizadora. Fonoaudiologia: informação para formação. Rio de Janeiro: Guanabara Koogan; 2003. p.55-65.

doi: 10.1590/S1516-18462010005000081

RECEBIDO EM: 04/07/2009

ACEITO EM: 21/04/2010

Endereço para correspondência:

Patrícia Reis Ferreira

Av Artur Bernardes, 84 ap. 101

Belo Horizonte - MG

CEP: 30350-310

E-mail patriciareisf@gmail.com 